\title{
Surface bound states and spin currents in non-centrosymmetric superconductors
}

\author{
A. B. Vorontsov,, , I. Vekhter, ${ }^{1}$ and M. Eschrig ${ }^{2}$ \\ ${ }^{1}$ Department of Physics and Astronomy, Louisiana State University, Baton Rouge, Louisiana, 70803, USA \\ ${ }^{2}$ Institut für Theoretische Festkörperphysik and DFG-Center for Functional Nanostructures, \\ Universität Karlsruhe, D-76128 Karlsruhe, Germany
}

(Dated: April 15, 2008)

\begin{abstract}
We investigate the influence of spin-orbit coupling in a non-centrosymmetric superconductor on its ground state properties near a surface. We determine the spectrum of Andreev bound states due to surface-induced mixing of bands with opposite spin helicities for a Rashba-type spin-orbit coupling. We find a qualitative change of the Andreev spectrum when we account for the suppression of the order parameter near the surface, leading to clear signatures in the surface density of states. We also compute the spin current at the surface, which has spin polarization normal to that of the bulk current. The magnitude of the current at the surface is enhanced in the normal state compared to the bulk, and even further enlarged in the superconducting phase. The particle and hole coherence amplitudes show Faraday-like rotations of the spin along quasiparticle trajectories.

PACS numbers: 72.25.-b, 74.45.+c, 73.20.At
\end{abstract}

The role of chirality and spin-orbit coupling in materials and nanostructures is a very active and promising subject in the fields of spintronics, superconductivity and magnetism [1, 2, 3]. The unusual properties of non-centrosymmetric (NCS) materials originate from the crystal structure that lacks a center of inversion, allowing for pronounced spin-orbit (SO) coupling that is odd in the electron momentum, and leading to a chiral ground state. The resulting two-band nature of NCS metals leads to effects reminiscent of semiconductor physics, such as birefringence and spin polarization of the electron wavepacket [4]. Especially promising is the presence of charge-neutral spin currents in the ground state [5, 6].

Since understanding of interface physics is one of the foundations for all potential applications, it is of pivotal interest to investigate how the physical properties of NCS materials are modified near surfaces. The key observation is that scattering events off interfaces in materials with strong spin-orbit effects are typically spin-active. Spins dominate the surface physics, and any successful theoretical treatment must take this into account.

The recently discovered class of NCS superconductors [7, 8, 9, 10] combines the strong SO coupling that governs the metallic bands with a non-trivial, chiral, spin structure of the superconducting (SC) order parameter [11, 12, 13]. As a result, one may expect that spin transport in the SC phase exhibits novel features compared to superconductors with negligible SO interaction. These features are expected to be especially prominent near surfaces and interfaces, where the physics is controlled by the Andreev bound states (ABS), built as a result of particle-hole coherent scattering. ABS are crucially important in unconventional superconductors [14], where the phase variation of the order parameter $(\mathrm{OP})$ on the Fermi surface [15] and the pairbreaking near interfaces may lead to a midgap peak in the density of states (DOS) at the surface. The ABS states control thermodynamic properties and stability of the surface phases 16, 17], and govern transport across interfaces [18, 19, 20].

In this Letter we study the Andreev states and spin currents at the surface of a NCS superconductor. We show that (i) the ABS spectrum is qualitatively modified by the self consistent suppression of the order parameter; (ii) the spin current is (a) strongly enhanced near the surface in the normal state; (b) further enhanced in the SC phase. We develop a detailed theory of these effects.

For a non-centrosymmetric material it is convenient to perform a canonical transformation from a spin basis (with fermion annihilation operators $c_{\mathbf{k} \mu}$ for spin $\mu=\uparrow, \downarrow$ ) to the so-called helicity basis ( $b_{\mathbf{k} s}$ with helicity $s= \pm$ ), that diagonalizes the kinetic part of the Hamiltonian,

$$
\mathcal{H}_{k i n}=\sum_{\mathbf{k} \mu \nu} c_{\mathbf{k} \mu}^{\dagger}\left(\xi_{\mathbf{k}}+\alpha \mathbf{g}_{\mathbf{k}} \boldsymbol{\sigma}\right)_{\mu \nu} c_{\mathbf{k} \nu}=\sum_{\mathbf{k} s} \varepsilon_{\mathbf{k} s} b_{\mathbf{k} s}^{\dagger} b_{\mathbf{k} s} .
$$

Here, $\xi_{\mathbf{k}}$ is the band dispersion relative to the chemical potential in the absence of SO interaction, $\alpha$ is the SO coupling strength, $\sigma$ is the vector of Pauli matrices, and $\mathbf{g}_{\mathbf{k}}$ is a normalized (see below) SO vector [11, 12] that is odd in momentum, $\mathbf{g}_{-\mathbf{k}}=-\mathbf{g}_{\mathbf{k}}$, see Fig. 1 The helicity band dispersion is $\varepsilon_{\mathbf{k} \pm}=\xi_{\mathbf{k}} \pm \alpha\left|\mathbf{g}_{\mathbf{k}}\right|$. SO interaction fixes the orientation of the quasiparticle spin with respect to its momentum in each helicity band.

The Hamiltonian (11) is time reversal invariant but lifts the spin degeneracy. The transformation from spin to helicity basis, $U_{\mathbf{k}}$, is defined by $U_{\mathbf{k}}\left(\mathbf{g}_{\mathbf{k}} \boldsymbol{\sigma}\right) U_{\mathbf{k}}^{\dagger}=\left|\mathbf{g}_{\mathbf{k}}\right| \sigma_{3}$, and determined by the direction of the $\mathbf{g}$-vector in $\mathbf{k}$ space,

$$
U_{\mathbf{k}}=e^{-i \frac{\theta_{\mathbf{g}}}{2} \mathbf{n}_{\mathbf{g}} \boldsymbol{\sigma}}, \quad \mathbf{n}_{\mathbf{g}}=\frac{\mathbf{g}_{\mathbf{k}} \times \hat{\mathbf{z}}}{\left|\mathbf{g}_{\mathbf{k}} \times \hat{\mathbf{z}}\right|},
$$

where $\hat{\mathbf{z}}$ is the unit vector in z-direction, and $\theta_{\mathbf{g}}$ is the polar angle between $\mathbf{g}_{\mathbf{k}}$ and $\hat{\mathbf{z}}[12]$.

To describe superconductivity we use the NambuGor'kov formalism modified for a helical basis. We define 


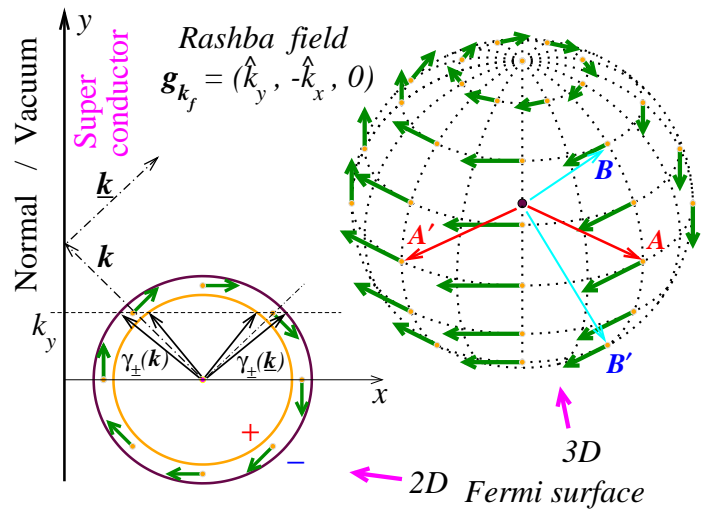

FIG. 1: (Color online) A map of the spin-orbit vector in momentum space for the Rashba form $\mathbf{g}_{\mathbf{k}}=\hat{\mathbf{k}} \times \hat{\mathbf{z}}$. On reflection the spin orbit vector $\mathbf{g}_{\mathbf{k}}$ may change, e.g. from $A \rightarrow A^{\prime}$, or not, $B \rightarrow B^{\prime}$. The scattering geometry is shown on the left.

the helical counterpart, $\hat{B}_{\mathbf{k}}^{\dagger}=\left(b_{\mathbf{k}+}^{\dagger}, b_{\mathbf{k}-}^{\dagger}, b_{\mathbf{k}+}, b_{\mathbf{k}-}\right)$, to the Nambu spinor, $\hat{C}_{\mathbf{k}}^{\dagger}=\left(c_{\mathbf{k} \uparrow}^{\dagger}, c_{\mathbf{k} \downarrow}^{\dagger}, c_{\mathbf{k} \uparrow}, c_{\mathbf{k} \downarrow}\right)$, by

$$
\hat{B}_{\mathbf{k}}=\hat{U}_{\mathbf{k}} \hat{C}_{\mathbf{k}}, \quad \hat{U}=\left(\begin{array}{cc}
U & 0 \\
0 & U^{*}
\end{array}\right)
$$

and construct $4 \times 4$ retarded Green's functions in helicity basis, $\hat{G}_{\mathbf{k}_{1} \mathbf{k}_{2}}\left(t_{1}, t_{2}\right)=-i \theta\left(t_{1}-t_{2}\right)\left\langle\left\{\hat{B}_{\mathbf{k}_{1}}\left(t_{1}\right), \hat{B}_{\mathbf{k}_{2}}^{\dagger}\left(t_{2}\right)\right\}\right\rangle_{\mathcal{H}}$, where $\hat{B}(t)$ are Heisenberg operators, the braces denote an anticommutator, $\langle\ldots\rangle_{\mathcal{H}}$ is a grand canonical average, and $\theta$ is the usual step function.

Below we employ the quasiclassical method 21] for treating the inhomogeneous surface problem. In the materials of interest $\alpha\left|\mathbf{g}_{\mathbf{k}_{f}}\right| \ll E_{f}$ for any Fermi momentum $\mathbf{k}_{f}$, where $E_{f}$ is the Fermi energy. In addition, the superconducting energy scales (transition temperature $T_{c}$ and the gap $\Delta$ ) are much smaller than $E_{f}$. Under these conditions quasiparticles with different helicity but with the same $\hat{\mathbf{k}} \equiv \mathbf{k} /|\mathbf{k}|$ propagate coherently along a common classical trajectory (determined by the Fermi surface for $\alpha=0, \xi_{\mathbf{k}_{f}}=0$ ), over distances much longer than the Fermi wavelength. We normalize $\mathbf{g}_{\mathbf{k}},\left\langle\mathbf{g}_{\mathbf{k}_{f}}^{2}\right\rangle=1$, where $\langle\ldots\rangle$ denotes a Fermi surface average. The quasiclassical propagator is then obtained as $\hat{g}\left(\mathbf{k}_{f}, \mathbf{R}, \epsilon, t\right)=$ $\hat{\tau}_{3} \int d \xi_{\mathbf{k}} \int(d \mathbf{q})(d \tau) e^{i(\mathbf{q} \mathbf{R}+\epsilon \tau)} \hat{G}_{\mathbf{k}+\frac{\mathbf{q}}{2}, \mathbf{k}-\frac{\mathbf{q}}{2}}\left(t+\frac{\tau}{2}, t-\frac{\tau}{2}\right)$ where $\hat{\tau}_{3}$ is the Pauli matrix in the particle-hole space. Using $U_{-\mathbf{k}} U_{\mathbf{k}}^{\dagger}=i \mathbf{n}_{\mathbf{g}} \boldsymbol{\sigma}$ and the fermionic anticommutation relations for the $b$ and $b^{\dagger}$, we derive the fundamental symmetry relations for the $2 \times 2 \mathrm{Nambu}$ matrix components, $g\left(\epsilon, \mathbf{k}_{f}\right)_{22}=\left[\left(\mathbf{n}_{\mathrm{g}} \boldsymbol{\sigma}\right) g\left(-\epsilon,-\mathbf{k}_{f}\right)_{11}\left(\mathbf{n}_{\mathbf{g}} \boldsymbol{\sigma}\right)\right]^{*}$ and $g\left(\epsilon, \mathbf{k}_{f}\right)_{21}=\left[\left(\mathbf{n}_{\mathbf{g}} \boldsymbol{\sigma}\right) g\left(-\epsilon,-\mathbf{k}_{f}\right)_{12}\left(-i \sigma_{2}\right)\left(\mathbf{n}_{\mathbf{g}} \boldsymbol{\sigma}\right)\right]^{*}\left(i \sigma_{2}\right)$.

Standard procedure 21] yields the Eilenberger equation in helicity basis,

$$
\left[\varepsilon \hat{\tau}_{3}-\alpha \hat{v}_{S O}-\hat{\Delta}, \hat{g}\right]+i \mathbf{v}_{f} \boldsymbol{\nabla} \hat{g}=\hat{0}
$$

with normalization $\hat{g}^{2}=-\pi^{2} \hat{1}$. Here, $\varepsilon$ is the energy, $\hat{v}_{S O}=\left|\mathbf{g}_{\mathbf{k}_{f}}\right| \sigma_{3} \hat{\tau}_{3}$, and $\hat{\Delta}$ is the superconducting OP. The velocity renormalization of order $\alpha / E_{f} \ll 1$ is neglected. We choose a separable pairing interaction consistent with the form of the gap, and determine $\hat{\Delta}$ self consistently with $\hat{g}$. In NCS superconductors the OP is a mixture of spin singlet $\left(\Delta_{s}\right)$ and triplet $\left(\Delta_{t}\right)$ components [13, 22]. Assuming that the triplet component aligns with $\mathbf{g}_{\mathbf{k}}$, in real gauge it is given by,

$$
\hat{\Delta}=\mathcal{Y}\left(\mathbf{k}_{f}\right)\left[\Delta_{s}(\mathbf{R}) \hat{1}+\Delta_{t}(\mathbf{R}) \hat{v}_{S O}\left(\mathbf{k}_{f}\right)\right]\left(i \sigma_{2}\right) \hat{\tau}_{1},
$$

where the basis function $\mathcal{Y}\left(\mathbf{k}_{f}\right)$ transforms according to one of the irreducible representations of the crystal point group, and $\left\langle\mathcal{Y}^{2}\left(\mathbf{k}_{f}\right)\right\rangle=1$. With the gap functions in the helicity bands, $\Delta_{ \pm}=\Delta_{s} \pm \Delta_{t}\left|\mathbf{g}_{\mathbf{k}_{f}}\right|$, the order parameter is $\Delta=\left\{\Delta_{+} \sigma^{+}-\Delta_{-} \sigma^{-}\right\} \mathcal{Y}$, where $\sigma^{ \pm}=\left(\sigma_{1} \pm i \sigma_{2}\right) / 2$.

We parameterize the Green's function by the coherence functions for particles and holes, $\gamma$ and $\bar{\gamma}(2 \times 2$ spin matrices), which allow a very intuitive physical interpretation of the Andreev scattering processes [23],

$\hat{g}=-i \pi\left(\begin{array}{cc}1-\gamma \bar{\gamma} & 0 \\ 0 & 1-\bar{\gamma} \gamma\end{array}\right)^{-1}\left(\begin{array}{cc}1+\gamma \bar{\gamma} & 2 \gamma \\ -2 \bar{\gamma} & -1+\bar{\gamma} \gamma\end{array}\right)$.

Fundamental symmetry relates $\gamma$ and $\bar{\gamma}$ in helicity basis by $\bar{\gamma}\left(\epsilon, \mathbf{k}_{f}\right)=\left[\left(\mathbf{n}_{\mathbf{g}} \boldsymbol{\sigma}\right) \gamma\left(-\epsilon,-\mathbf{k}_{f}\right)\left(-i \sigma_{2}\right)\left(\mathbf{n}_{\mathbf{g}} \boldsymbol{\sigma}\right)\right]^{*}\left(i \sigma_{2}\right)$. In the bulk, $\gamma=\gamma_{+}^{0} \sigma^{+}-\gamma_{-}^{0} \sigma^{-}$, and $\bar{\gamma}=\tilde{\gamma}_{-}^{0} \sigma^{+}-\tilde{\gamma}_{+}^{0} \sigma^{-}$, with $\gamma_{ \pm}^{0}\left(\epsilon, \mathbf{k}_{f}\right)=-\Delta_{ \pm}\left(\mathbf{k}_{f}\right) /\left(\epsilon+i \sqrt{\left|\Delta_{ \pm}\left(\mathbf{k}_{f}\right)\right|^{2}-\epsilon^{2}}\right)$ and $\tilde{\gamma}_{ \pm}^{0}\left(\mathbf{k}_{f}, \epsilon\right)=\gamma_{ \pm}^{0}\left(-\mathbf{k}_{f},-\epsilon\right)^{*}$.

The surface bound states are determined by the poles of the Green's function, Eq. (6). We consider specular reflection, whereby the component of $\mathbf{k}$ normal to surface changes sign, $\mathbf{k} \rightarrow \underline{\mathbf{k}}$, see Fig. 1. We find the amplitudes $\gamma_{\mathbf{k}}\left(\bar{\gamma}_{\mathbf{k}}\right)$, by integrating forward (backward) along incoming, $\mathbf{k}$, (outgoing, $\underline{\mathbf{k}}$ ) trajectory starting from the values in the bulk 23]. The amplitudes $\gamma_{\underline{\mathbf{k}}}$ and $\bar{\gamma}_{\mathbf{k}}$, in contrast, are determined from the boundary conditions at the surface. Since the surface is non-magnetic, the components of $\hat{g}$ in the spin basis, $\hat{g}_{\mathbf{k}}^{\mathrm{s}}=\hat{U}_{\mathbf{k}}^{\dagger} \hat{g}_{\mathbf{k}} \hat{U}_{\mathbf{k}}$, are continuous at the surface. This leads to a surface induced mixing of the helicity bands according to $U_{\underline{\mathbf{k}}}^{\dagger} \gamma_{\underline{\mathbf{k}}} U_{\underline{\mathbf{k}}}^{*}=\gamma_{\underline{\mathbf{k}}}^{\mathrm{s}}=\gamma_{\mathbf{k}}^{\mathrm{s}}=U_{\mathbf{k}}^{\dagger} \gamma_{\mathbf{k}} U_{\mathbf{k}}^{*}$, and $U_{\mathbf{k}}^{T} \bar{\gamma}_{\mathbf{k}} U_{\mathbf{k}}=\bar{\gamma}_{\mathbf{k}}^{s}=\bar{\gamma}_{\underline{\mathbf{k}}}^{\mathrm{s}}=U_{\underline{\mathbf{k}}}^{T} \bar{\gamma}_{\underline{\mathbf{k}}} U_{\mathbf{k}}$. From Eq. (何), the bound states correspond to the zero eigenvalues of the matrix $1-\gamma_{\mathbf{k}} \bar{\gamma}_{\mathbf{k}}=1-\gamma_{\mathbf{k}} U_{\mathbf{k}}^{*} U_{\underline{\mathbf{k}}}^{T} \bar{\gamma}_{\underline{\mathbf{k}}} U_{\underline{\mathbf{k}}} U_{\mathbf{k}}^{\dagger}$ at the surface, and we derive our final equation for the ABS energies via the surface amplitudes in the helicity basis

$$
\left(1+\gamma_{+} \tilde{\gamma}_{+}\right)\left(1+\gamma_{-} \tilde{\gamma}_{-}\right)=-\left(1+\gamma_{+} \tilde{\gamma}_{-}\right)\left(1+\gamma_{-} \tilde{\gamma}_{+}\right) \mathcal{M} .
$$

The "mixing" factor $\mathcal{M}$ is determined by the change of $\mathrm{g}_{\mathrm{k}}$ under reflection $\mathbf{k} \rightarrow \underline{\mathbf{k}}$ at the surface,

$$
\mathcal{M}=\frac{\sin ^{2} \frac{\theta_{\mathbf{g}}-\theta_{\underline{\underline{g}}}}{2}+\sin ^{2} \frac{\theta_{\mathbf{g}}+\theta_{\underline{\underline{g}}}}{2} \tan ^{2} \frac{\phi_{\mathbf{g}}-\phi_{\underline{\underline{g}}}}{2}}{\cos ^{2} \frac{\theta_{\mathbf{g}}-\theta_{\mathbf{g}}}{2}+\cos ^{2} \frac{\theta_{\mathbf{g}}+\theta_{\mathbf{g}}}{2} \tan ^{2} \frac{\phi_{\mathbf{g}}-\phi_{\mathbf{g}}}{2}},
$$

where $\theta_{\mathbf{g}}, \phi_{\mathbf{g}}$ and $\theta_{\underline{\mathbf{g}}}, \phi_{\underline{\mathbf{g}}}$ are the polar and azimuthal angles of $\mathbf{g}_{\mathbf{k}}$ and $\mathbf{g}_{\underline{\mathbf{k}}}$, respectively. If $\mathbf{g}_{\mathbf{k}}=\mathbf{g}_{\underline{\mathbf{k}}}\left(B \rightarrow B^{\prime}\right.$ in Fig. (1) there is no helicity band mixing, $\overline{\mathcal{M}}=0$, and we 

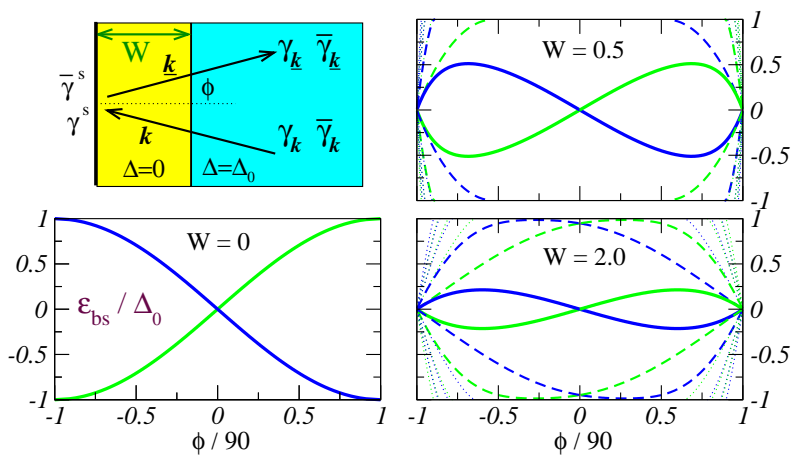

FIG. 2: (Color online) Bound state energy as a function of the impact angle for different widths $W$ (in units of of $v_{f} / 2 \Delta_{0}$ ) of the order parameter suppression region. Blue (green) lines correspond to plus (minus) sign in Eq. (9). Solid lines: principal mode; broken lines: higher multiple reflection modes.

recover the conditions for $\mathrm{ABS}$ in superconductors with no SO coupling. The limit $\mathcal{M} \rightarrow \infty$ describes pure interband scattering. In the general case $\left(A \rightarrow A^{\prime}\right.$ in Fig. 1) a finite $\mathcal{M}$ determines the relative weights of intraband and interband scattering.

While assuming a uniform OP up to the surface to obtain the ABS spectra may seem reasonable, we show now that the suppression of the anisotropic (triplet) component of the OP in Eq. (5) near the surface drastically modifies the ABS spectrum and the surface DOS, $N\left(\varepsilon, \mathbf{k}_{f}\right)=-\frac{N_{f}}{2 \pi} \operatorname{Im} \operatorname{Tr}\left\{g\left(\varepsilon, \mathbf{k}_{f}\right)\right\}$, where $\operatorname{Tr}$ is a $2 \times 2$ spin trace, and $N_{f}$ is the normal state DOS. Hereafter we consider a $2 \mathrm{D}$ material with the Rashba type SO coupling $\alpha=\alpha_{R} k_{f}, \mathbf{g}_{\mathbf{k}}=(\mathbf{k} \times \hat{\mathbf{z}}) / k_{f}$, and a triplet order parameter, $\Delta_{+}=-\Delta_{-}=\Delta$; results for $\Delta_{+} \neq-\Delta_{-}$and different SO couplings will be presented elsewhere [24].

To obtain insight in the role of the OP suppression, we consider first a simple model where $\Delta=0$ in a layer of width $W$ next to the surface, see Fig. 2. Trajectories incident at an angle $\phi$ travel through a non-SC region of an effective width $2 D=2 W / \cos \phi$. In this case $\mathcal{M}=\cot ^{2} \phi$, the surface coherence amplitudes gain a phase factor, $\gamma_{ \pm}=\gamma_{ \pm}^{0} e^{i 2 \varepsilon D / v_{f}}, \tilde{\gamma}_{ \pm}=\tilde{\gamma}_{ \pm}^{0} e^{i 2 \varepsilon D / v_{f}}$, and the bound states are given by $\operatorname{Im}^{2}\left(\tilde{\gamma}_{+}^{0} e^{i 2 \varepsilon D / v_{f}}\right)=$ $\operatorname{Re}^{2}\left(\tilde{\gamma}_{+}^{0} e^{i 2 \varepsilon D / v_{f}}\right) \mathcal{M}$, which yields

$$
\frac{\varepsilon}{\sqrt{\Delta_{0}^{2}-\varepsilon^{2}}}=-\tan \left(\frac{2 W \varepsilon}{v_{f} \cos \phi} \pm \phi\right) \text {. }
$$

Solutions of this equation are shown in Fig. 2. The "principal" modes with energies away from the continuum edge contribute the most to the subgap DOS. $W=0$ reproduces the result of Ref. 25: each incoming trajectory yields a bound state at a different energy. For $W \neq 0$ the main mode $\varepsilon_{b s}(\phi)$ develops a maximum at $\varepsilon^{\star}<\Delta_{0}$, and we expect a peak in the surface DOS near $\varepsilon^{\star}$ due to abundance of trajectories contributing to $N\left(\varepsilon^{\star}\right)$.

Fully self-consistent solution, shown in Fig. 3(b), con-
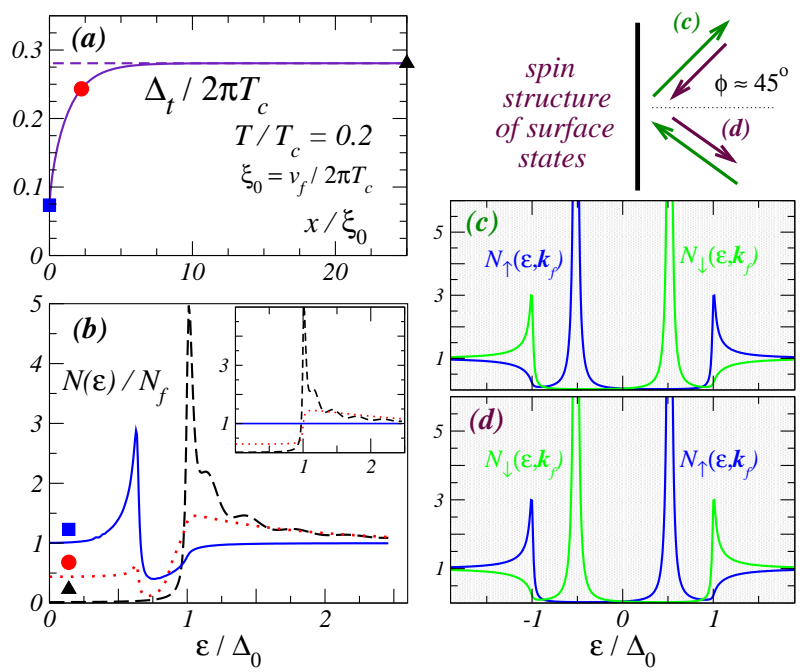

FIG. 3: (Color online) Structure of the surface states for a Rashba triplet superconductor $\left(\Delta_{+}=-\Delta_{-}\right)$. (a) Order parameter suppression; (b) DOS at locations indicated in (a); the large sub-gap peaks are due to the suppression of $\Delta$. Inset: DOS for a homogeneous order parameter; note the absence of any subgap features. (c,d) spin-resolved surface DOS for two trajectories; $N_{\uparrow}\left(\epsilon, \mathbf{k}_{f}\right)$ (blue) and $N_{\downarrow}\left(\epsilon, \mathbf{k}_{f}\right)$ (green) correspond to blue and green branches in Fig. 2 .

firms this. Note that $\Delta \neq 0$ at the surface, Fig. 3(a), as in other unconventional superconductors misaligned with respect to the interface [16]. Crucially, self-consistency does yield a peak in the surface DOS below the gap at a finite energy. Experimentally accessing this peak by point contact tunneling requires a sufficiently wide tunneling cone as the feature arises from the trajectories at intermediate incident angles, see Fig. 2.

These ABS have unusual spin structure. Fig. $3(\mathrm{c}, \mathrm{d})$ shows the spin resolved density of states, $N_{\uparrow \downarrow}=N \pm$ $N^{Z}$, where $N$ is the net DOS and $N^{\alpha}\left(\epsilon, \mathbf{k}_{f}, \mathbf{x}\right)=$ $-\frac{N_{f}}{2 \pi} \operatorname{Im} \operatorname{Tr}\left\{\sigma^{\alpha} g\left(\epsilon, \mathbf{k}_{f}, \mathbf{x}\right)\right\}$. At the interface $N^{X}=N^{Y}=$ 0 . The states corresponding to different branches of Eq. (9) have opposite spin polarization. Since the spin polarization changes sign for reversed trajectories, the Andreev states carry spin current along the interface.

Spin currents exist in NCS materials because the spin is not conserved, and consequently precession terms enter the continuity equation, $\partial_{t} S^{\alpha}(\mathbf{x})+\boldsymbol{\nabla} \cdot \boldsymbol{\Pi}^{\alpha}(\mathbf{x})=P^{\alpha}(\mathbf{x})[5]$. Here, the spin density, $S^{\alpha}(\mathbf{x})=\frac{1}{2} \operatorname{Tr} \int d \mathbf{k} \sigma^{\alpha} G(\mathbf{k}, \mathbf{x})$, the spin current, $\boldsymbol{\Pi}^{\alpha}(\mathbf{x})=\frac{1}{4} \operatorname{Tr} \int d \mathbf{k}\left\{\sigma^{\alpha}, \mathbf{v}_{\mathbf{k}}\right\} G(\mathbf{k}, \mathbf{x})$, and the precession $P^{\alpha}(\mathbf{x})=\frac{1}{2 i} \operatorname{Tr} \int d \mathbf{k}\left[\sigma^{\alpha}, \mathbf{v}_{\mathbf{k}} \cdot \mathbf{k}\right] G(\mathbf{k}, \mathbf{x})$, (where $[\bullet, \bullet]$ is a commutator, and $\mathbf{v}_{\mathbf{k}}=\mathbf{k}_{f} / m+\alpha_{R}(\hat{\mathbf{z}} \times \boldsymbol{\sigma})$ is the band velocity), are all given in terms of Green's functions at imaginary relative time $\tau=-i 0$. For the Rashba case, the precession terms are related to spin currents via the relations $P^{X}=-2 m \alpha_{R} \Pi_{x}^{Z}, P^{Y}=$ $-2 m \alpha_{R} \Pi_{y}^{Z}, P^{Z}=2 m \alpha_{R}\left(\Pi_{x}^{X}+\Pi_{y}^{Y}\right) \cdot[26]$

We first consider the spin currents in the normal state. The bulk value, $\Pi_{x}^{Y}=-\Pi_{y}^{X}=\Pi_{\text {spin }}^{\text {bulk }}=m^{2} \alpha_{R}^{3} / 3 \pi$ agrees 

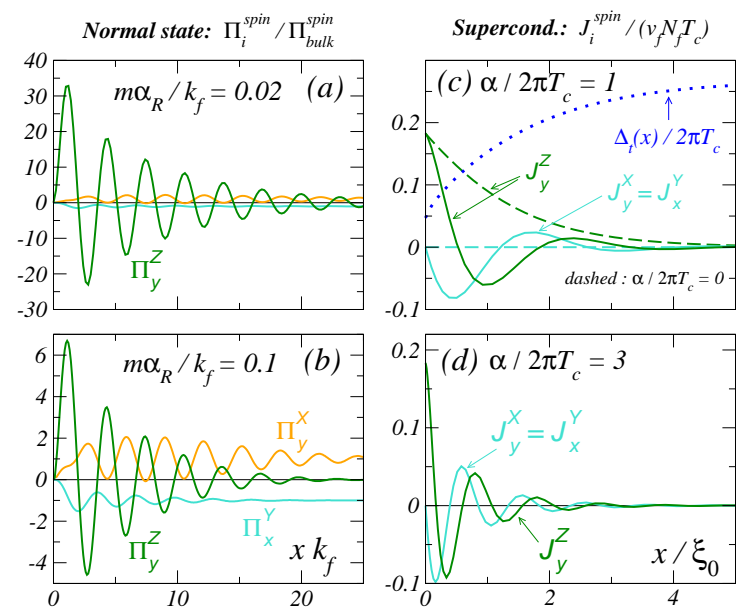

FIG. 4: (Color online) Spin currents near the surface. Left: Rashba metal. The non-vanishing components in the bulk are $\Pi_{x}^{Y}=-\Pi_{y}^{X}$. Near the surface $\Pi_{y}^{Z}$ is large, see text. Right: Spin currents and the order parameter in the SC state.

with Ref. 5. To determine the surface spin currents we find the Green's function for a surface modeled as a $\delta$ function barrier at $x=0$ of strength $U$. The Dyson equation in $2 \times 2$ spin space reads $G^{-1}=\left[G^{(0)}\right]^{-1}-U \delta(x)$, where $\left[G_{\mathbf{k}}^{(0)}\right]^{-1}=\varepsilon-\xi_{\mathbf{k}}-\alpha_{R}(\mathbf{k} \times \hat{\mathbf{z}}) \boldsymbol{\sigma}$. For an impenetrable surface $(U \rightarrow \infty)$ the solution is (for fixed $\left.k_{y}\right)$ 27]

$$
G_{k_{x} k_{x}^{\prime}}=G_{k_{x}}^{(0)} 2 \pi \delta\left(k_{x}-k_{x}^{\prime}\right)-G_{k_{x}}^{(0)} \frac{1}{\int \frac{d p_{x}}{2 \pi} G_{p_{x}}^{(0)}} G_{k_{x}^{\prime}}^{(0)} .
$$

We solve Eq. (10) numerically, and show the normal state surface spin currents in Fig. 4(a,b). The most prominent new feature is a large surface current $\Pi_{y}^{Z}$ with out of plane spin polarization (reminiscent to that in spin Hall bars [28]) that flows along the surface, and decays rapidly into the bulk on a Fermi wavelength scale. This component is related to $\Pi_{x}^{Y}$ via the continuity equation, $\Pi_{y}^{Z}(x)=$ $-1 /\left(2 m \alpha_{R}\right) d \Pi_{x}^{Y}(x) / d x$. As a result, this component is much greater, by a factor of order $k_{f} / m \alpha_{R}$, than the bulk spin currents in the normal state.

The SC spin current, shown in Fig. 4( c,d), is defined in the quasiclassical method relative to the normal state,

$$
\mathbf{J}^{\alpha} \equiv \mathbf{\Pi}^{\alpha}-\mathbf{\Pi}_{N}^{\alpha}=\int_{-\infty}^{\infty} d \epsilon n_{f}(\epsilon)\left\langle\mathbf{v}_{f} N^{\alpha}\left(\epsilon, \mathbf{k}_{f}, \mathbf{x}\right)\right\rangle
$$

where $n_{f}(\epsilon)$ is the Fermi function. The surface-induced current with out of plane spin polarization is greater than the normal state current by the factor $\sim T_{c} E_{f}^{2} / \alpha^{3}$. The maximal amplitude at the surface is solely determined by the structure of the SC gap and formally survives even in the limit $\alpha \rightarrow 0$. SC spin currents decay into the bulk on the scale of the coherence length, much slower than in the normal phase. The oscillations in Fig. 廿( $(\mathrm{c}, \mathrm{d})$ are determined by the spin-orbit strength $\alpha$ and appear due to Faraday-like rotations of the spin coherence functions along quasiparticle trajectories.
In summary, we developed a framework for the analysis of surface bound states and the associated spin currents in non-centrosymmetric superconductors, and applied it to a system with a Rashba-type spin-orbit coupling. We found that the suppression of superconductivity near the surface gives rise to a finite bias peak in the surface density of states that can be probed by point contact tunneling. We also showed that large in amplitude and slowly decaying spin currents with out of plane spin polarization are carried by these surface states. This opens the route to future investigations of spin transport in systems containing superconductors without center of inversion.

Acknowledgements. This work was supported by the Louisiana Board of Regents, and through I2CAM by NSF grant DMR 0645461.

* Present address: Dept. of Physics, University of Wisconsin, Madison, WI 53706

[1] M. Bode et al., Nature 447, 190 (2007); C. Pfleiderer et al., Nature 427, 227 (2004);

[2] L. Meier et al., Nature Physics 3, 650 (2007);

[3] T. Champel, T. Löfwander, M. Eschrig, Phys. Rev. Lett. 100, 077003 (2008).

[4] M. Khodas, A. Shekhter, and A. Finkel'stein, Phys. Rev. Lett. 92, 086602 (2004); V. M. Ramaglia et al., J. Phys. Cond. Matt. 16, 9143 (2004).

[5] E. I. Rashba, Phys. Rev. B 68, 241315 (2003).

[6] S. Murakami, N. Nagaosa, and S.-C. Zhang, Science 301, 1348 (2003).

[7] E. Bauer et al., Phys. Rev. Lett. 92, 027003 (2004).

[8] H. Q. Yuan et al., Phys. Rev. Lett. 97, 017006 (2006).

[9] T. Akazawa et al., J. of Phys.: Cond. Matt. 16, L29 (2004).

[10] K. Sugawara et al., Phys. Rev. B 76, 132512 (2007).

[11] L. P. Gor'kov and E. I. Rashba, Phys. Rev. Lett. 87, 037004 (2001).

[12] P.A.Frigeri et al., Phys. Rev. Lett. 92, 097001 (2004).

[13] P.A.Frigeri et al., Europ. Phys. Journ. B 54, 435 (2006).

[14] T. Löfwander, V. S. Shumeiko, and G. Wendin, Supercond. Sci. Technol. 14, R53 (2001).

[15] M. Sigrist and K. Ueda, Rev. Mod. Phys. 63, 239 (1991).

[16] L. Buchholtz et al., J. Low Temp. Phys. 101, 1079 (1995).

[17] M. Matsumoto and H. Shiba, J. Phys. Soc. Jpn. 64, 4867 (1995).

[18] M. Covington et al., Phys. Rev. Lett. 79, 277 (1997).

[19] M.Aprili et al., Phys. Rev. B 57, R8139 (1998).

[20] M. Fogelström, D. Rainer, and J. A. Sauls, Phys. Rev. Lett. 79, 281 (1997).

[21] J. W. Serene and D. Rainer, Phys. Rep. 101, 221 (1983).

[22] I. A. Sergienko and S. H. Curnoe, Phys. Rev. B 70, 214510 (2004).

[23] M. Eschrig, Phys. Rev. B 61, 9061 (2000).

[24] A. B. Vorontsov et al., (unpublished).

[25] C. Iniotakis et al., Phys. Rev. B 76, 012501 (2007).

[26] S. I. Erlingsson, J. Schliemann, and D. Loss, Phys. Rev. B 71, 035319 (2005). Note that our last equation differs from Eq.(33) of this paper.

[27] M. Matsumoto and H. Shiba, J. Phys. Soc. Jpn. 64, 1703 (1995).

[28] E. G. Mishchenko, A. V. Shytov, and B. I. Halperin, Phys. Rev. Lett. 93, 226602 (2004). 